\title{
Combined shockwave intravascular lithotripsy and ultrahigh-pressure balloon dilatation for the treatment of stent underexpansion secondary to severe coronary calcification
}

\author{
Andreas S. Kalogeropoulos ${ }^{1,2}$, Grigoris V. Karamasis ${ }^{3}$, Antonis N. Pavlidis², \\ Nasos Papadothomakos ${ }^{1}$, Eleftherios Sakadakis', Panos Vardas¹, Stylianos Tzeis ${ }^{1}$ \\ 1 Department of Cardiology, MITERA General Hospital, Hygeia Group, Athens, Greece \\ 2 Department of Cardiology, St Thomas' Hospital, London, United Kingdom \\ 3 Department of Cardiology, Essex Cardiothoracic Center, Basildon, United Kingdom
}

Correspondence to: Andreas S. Kalogeropoulos, MD, PhD, MRCP, Department of Cardiology, MITERA General Hospital, Erythrou Stavrou 6, 15123 Athens, Greece, phone: +302106869777, email: andkalog@gmail.com Received: November 4, 2020. Revision accepted: January 4, 2021. Published online: January 18, 2021. Kardiol Pol. 2021; 79 (2): 205-206 doi:10.33963/KP.15753 Copyright by the Author(s), 2021
A 70-year-old woman treated with percutaneous coronary intervention (PCI) of the left anterior descending artery (LAD) with $2.5 \times 29-\mathrm{mm}$ and $2.5 \times 18$-mm FIREHAWK drug-eluting stents (Microport Medical, Shanghai, China) 6 months previously was admitted to our center for the treatment of recurrent angina pectoris. Transradial coronary angiography showed mid-LAD stent underexpansion with significant distal stenosis (FIGURE 1A; Supplementary material, Video S1). Intravascular ultrasound (IVUS)-guided PCI of the LAD was performed. The left main coronary artery was cannulated with a 6 Fr Convey LEFT BU3.5 guiding catheter (Boston Scientific, Marlborough, Massachusetts, United States). A SION blue guidewire (ASAHI INTECC Co., Ltd., Aichi, Japan) was placed distally. Using a Guidezilla-2 catheter extension (Boston Scientific), we advanced an OptiCross IVUS catheter (Boston Scientific) distally to the stents. Intravascular ultrasound demonstrated serial segments of stent underexpansion with nodular calcification and strut malapposition, which were not visualized angiographically (FIGURE 1B-1D; Supplementary material, Video S2). We evaluated the distal lesion angiographically only, due to the inability of advancing the IVUS catheter to the distal LAD.

We proceeded with stent dilatation with a $2.75 \times 12-\mathrm{mm}$ noncompliant balloon at 20 atm (Supplementary material, Figure S1) followed by intravascular lithotripsy (IVL) (Shockwave
Medical, Fremont, California, United States) with a $3 \times 12-\mathrm{mm}$ balloon. After balloon inflation at 4 to 6 atm, 8 cycles of 10 pulses of circumferential energy each were delivered along the underexpanded stent segments (Supplementary material, Figure S2A-S2C). Angiography demonstrated persistent stent wasting likely secondary to eccentric calcification and stent recoil (Supplementary material, Video S3). We therefore performed dilatation with a $3 \times 10$-mm ultrahigh-pressure OPN balloon (SIS Medical AG, Winterthur, Switzerland) at 40 atm (Supplementary material, Figure $S 2 D-2 F$ ). Another IVUS evaluation demonstrated significant improvement of stent expansion with areas of fragmented calcification and elimination of malapposition (FIGURE 1E-1F, Supplementary material, Video S4). Additionally, stent dilatations with $3 \times 15-\mathrm{mm}$ and $3 \times 20$-mm drug-eluting balloons were successfully performed (Supplementary material, Figure $S 2 G-S 2 H$ ). The distal lesion was predilated with a $2 \times 20$-mm noncompliant balloon, followed by final dilatation with a $2 \times 30$-mm drug eluting balloon with an excellent final angiographic result (Supplementary material, Figure S1I and Video S5).

Extensive plaque calcification may adversely impact PCI by compromising effective dilatation of coronary stenosis and increase the likelihood of stent underexpansion and failure. ${ }^{1} \mathrm{Un}$ til now, undilatable lesions in previously stented segments have been conventionally approached 

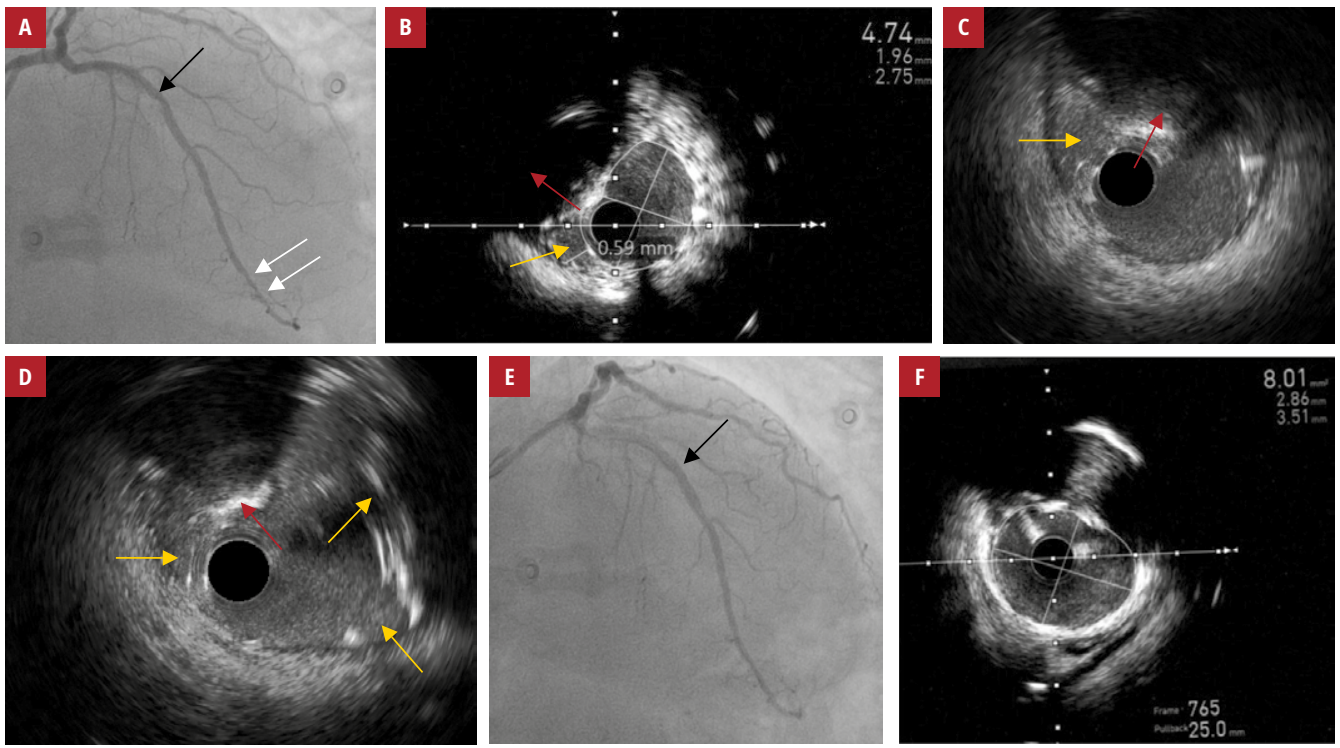

FIGURE 1 A - coronary angiography of the left anterior descending artery in the anteroposterior cranial projection visualizing stent underexpansion (black arrow) and significant distal stenosis (white arrows); B-D - intravascular ultrasound (IVUS) demonstrating severe eccentric nodular calcification (red arrows) and confirming the angiographically shown underexpansion of the stent (minimum stent area [MSA] at the underexpanded segment of the stent, $4.74 \mathrm{~mm}^{2}$; with a reference cross-sectional area of $7.57 \mathrm{~mm}^{2}$ and a reference diameter of 2.87-3.28 mm indicating significantly reduced relative expansion of 63\%; panel B). IVUS showed additional areas of stent underexpansion (panels $\mathbf{C}$ and $\mathbf{D}$ ) not visualized in the coronary angiography, and strut malapposition (malapposition distance, $590 \mu \mathrm{m}$; panels B-D, yellow arrows); E - final coronary angiography showing improvement of the underexpanded stent area (black arrow); F - IVUS showing significant improvement of stent expansion (MSA, $8.01 \mathrm{~mm}^{2}$; relative expansion, >100\%) and elimination of strut malapposition

with cutting balloons or laser and rotational atherectomy, with unpredictable results. ${ }^{2}$ The novel coronary IVL has shown encouraging results after being used to treat undilatable calcified lesions., ${ }^{3,4}$ Additionally, in severe coronary calcification, prompt use of IVL can prevent potentially catastrophic PCI complications. ${ }^{5}$

In our case, a novel approach was applied to treat underexpanded stent due to superimposed nodular calcifications with the combined use of IVL and OPN balloons. The IVL system generated the energy to safely disrupt circumferential calcium, whilst the ultrahigh-pressure OPN balloon conveyed the force to appropriately expand the stent and correct malapposition in areas of eccentric calcification. This case illustrated the usefulness of IVUS in the treatment of stent underexpansion. In particular, IVUS aided to: 1) detect challenging lesions with nodular calcifications; 2) quantify stent underexpansion and strut malapposition; 3) define an effective PCI strategy.

\section{SUPPLEMENTARY MATERIAL}

Supplementary material is available at www.mp.pl/kardiologiapolska.

\section{ARTICLE INFORMATION}

CONFLICT OF INTEREST None declared.

OPEN ACCESS This is an Open Access article distributed under the terms of the Creative Commons Attribution-NonCommercial-NoDerivatives $4.0 \mathrm{In}$ ternational License (CC BY-NC-ND 4.0), allowing third parties to download articles and share them with others, provided the original work is properly cited, not changed in any way, distributed under the same license, and used for noncommercial purposes only. For commercial use, please contact the journal office at kardiologiapolska@ptkardio.pl.

HOW TO CITE Kalogeropoulos AS, Karamasis GV, Pavlidis AN, et al. Combined shockwave intravascular lithotripsy and ultrahigh-pressure balloon dilatation for the treatment of stent underexpansion secondary to severe coronary calcification. Kardiol Pol. 2021; 79: 205-206. doi:10.33963/KP.15753

\section{REFERENCES}

1 Raber L, Mintz GS, Koskinas KC, et al. Clinical use of intracoronary imaging. Part 1: guidance and optimization of coronary interventions. An expert consensus document of the European Association of Percutaneous Cardiovascular Interventions. EuroIntervention. 2018; 14: 656-677.

2 Latib A, Takagi K, Chizzola G, et al. Excimer Laser LEsion modification to expand non-dilatable stents: the ELLEMENT registry. Cardiovasc Revasc Med. 2014; 15: 8-12.

3 Tomasiewicz B, Kosowski M, Zimoch W, et al. Heavily calcified coronary lesion treated by shockwave intravascular lithotripsy. Kardiol Pol. 2019; 77: 890-891.

4 Legutko ], Niewiara L, Tomala M, et al. Successful shockwave intravascular lithotripsy for a severely calcified and undilatable lefty anterior descending coronary artery lesion in a patient with recurrent myocardial infarction. Kardiol Pol. 2019; 77: 723-725.

5 Iwańczyk S, Siniawski A, Panowicz M, et al. Successful intravscular lithotripsy for covered stent underexpansion due to severely calcified plaque. Kardiol Pol. 2020; 78: 247-248. 